\title{
Un Laboratorio de Ingeniería de Control Basado en Internet
}

Nourdine Aliane, Javier Fernández, Aída Martínez y Jaime Ortiz

Universidad Europea de Madrid, Departamento de Arquitectura de Computadores y Automática, C/ El bosque s/n, Villaviciosa de Odón, 28670 Madrid-España (e-mail: nourdine.aliane@uem.es)

\section{Resumen}

En este artículo se presenta el desarrollo de un laboratorio remoto basado en Internet para la enseñanza de la ingeniería de control desarrollado en la Universidad Europea de Madrid. El sistema ofrece la posibilidad de realizar experimentos de control en lazos abierto o cerrado con controladores predefinidos que se pueden ejecutar en el lado del servidor o del cliente. Las características más destacables del sistema son el acceso concurrente de hasta un máximo de cuatro usuarios, la generación automática de informes de los experimentos y la reutilización de plantillas de los experimentos. Finalmente se detalla la arquitectura del sistema implementado, describiendo sus funcionalidades más destacadas.

Palabras clave: laboratorios remotos, control remoto, Internet, ingeniería de control

\section{An Internet-Based Control Engineering Laboratory}

\begin{abstract}
This paper presents the development of an internet-based remote laboratory for control engineering teaching education developed at the Universidad Europea de Madrid. The system allows users to perform control experiments in open or closed loops, using predefined structure controllers that can be executed on the server side or in the client side. The main features of the remote system are concurrent access for up to four users, automatic report generation, and reusing templates of the experiments. Finally, the system architecture is detailed presenting and discussing its most important features.
\end{abstract}

Keywords: remote laboratories, remote control, internet, control engineering 


\section{INTRODUCCIÓN}

Un laboratorio remoto es un conjunto de equipos físicos que se pueden operar y controlar remotamente, utilizando una interfaz determinada (Dormido, 2004). Estos equipos pueden ser didácticos como las maquetas de laboratorio o equipos industriales como los que pueda tener un banco de pruebas. Estos laboratorios requieren de servidores específicos que gestionan tanto a los usuarios del sistema como los equipos integrados en dichos sistemas.

En los últimos años, la tecnología de los laboratorios remotos ha mostrado una actividad investigadora significativa. En este contexto, la Unión Europea ha financiado numerosos proyectos para impulsar el desarrollo de esta tecnología e investigar su impacto en la educación. CYBERLAB (Cyberlab, 2007) es el proyecto más significativos y ha culminado con la creación de un proveedor de servicios para la integración de laboratorios en red. PEARL (Cooper, 2003; 2005) es otro proyecto enfocado al desarrollo de infraestructuras que permita el acceso remoto a los equipos de un laboratorio e investigar su impacto pedagógico para validar su desarrollo. Finalmente, MARVEL (Müller y Ferreira, 2005) es otro proyecto de envergadura que se propone desarrollar un entorno mixto que combina laboratorios remotos y virtuales. Estos proyectos ponen de manifiesto el rol preponderante que van a tener los laboratorios remotos en la educación en un futuro próximo.

En el área del control automático, la idea de compartir los equipos de un laboratorio, con fines educativos, se remonta a principios de los noventa, y en el trabajo de Aburdene et al. (1991), se propone un esquema básico de acceso remoto a los equipos de un laboratorio y como compartirlos con otras universidades. En la literatura, el artículo de Aktan y Bohus (1996), se considera como el primer trabajo importante enfocado a la enseñanza del control automático a través de Internet, y desde entonces, se han llevado a la práctica varias implementaciones reales. Entre estas realizaciones, destacan el laboratorio de sistemas dinámicos del Instituto Tecnológico de Stevens (Nickerson et al., 2007; Esche, 2006; Aziz et al., 2006) y el laboratorio de control de la Universidad de Siena (Casini et al., 2004; Casini y Prattichizzo, 2003). Estos laboratorios ofrecen la posibilidad de experimentar sobre maquetas de control como el control de nivel, el control de temperatura o el control de servomotores.

En lo que se refiere a las soluciones tecnológicas adoptadas en el diseño de las aplicaciones software para la gestión de los laboratorios remotos, el lector puede encontrar el buen estado de arte en varias publicaciones (Esche, 2006; Candelas y Moreno 2005; Bernstein y Ashrafioun, 2004; Dormido, 2004). Por otra parte, en la pagina Web del Comité Español de Automática (CEA-IFAC, 2007), el lector puede tener acceso a una recopilación de varios laboratorios virtuales y/o remotos relacionados con el área de Ingeniería de sistemas como la robótica, visión por computador, la automatización o el control automático.

En este artículo se presenta un laboratorio remoto basado en Internet para la educación en la ingeniería de control desarrollado en la Universidad Europea de Madrid. Este laboratorio permite realizar una gama extensa de experimentos de control. El sistema ha sido desarrollado con el doble objetivo: ofrecer a los estudiantes un entorno de experimentación remota transparente, y por otro lado, fácil de mantener por parte del equipo administrador. Actualmente, está siendo utilizado en una asignatura de ingeniería de control del plan de estudios de ingeniero en informática.

\section{DESCRIPCIÓN DEL SISTEMA}

El laboratorio remoto, denominado LABNET, tiene como objetivo la realización de experimentos de control sobre las maquetas de laboratorio a través de Internet. Actualmente, los usuarios pueden experimentar sobre tres modelos de maquetas: control de nivel, control de temperatura y un sistema de estabilización de barcos.

La arquitectura de LABNET sigue el modelo de cliente/servidor tal y como se muestra en la Fig. 1. El servidor es un ordenador personal con un procesador Pentium-III bajo Windows 2000, que está conectado, por un lado, a la red para dar servicio a los clientes, y por otro, a las maquetas de laboratorio por medio de dos tarjetas de adquisición de datos de tipo PC-LAB-816 para bus ISA. Estas dos tarjetas permiten la conexión de hasta cuatro maquetas. 


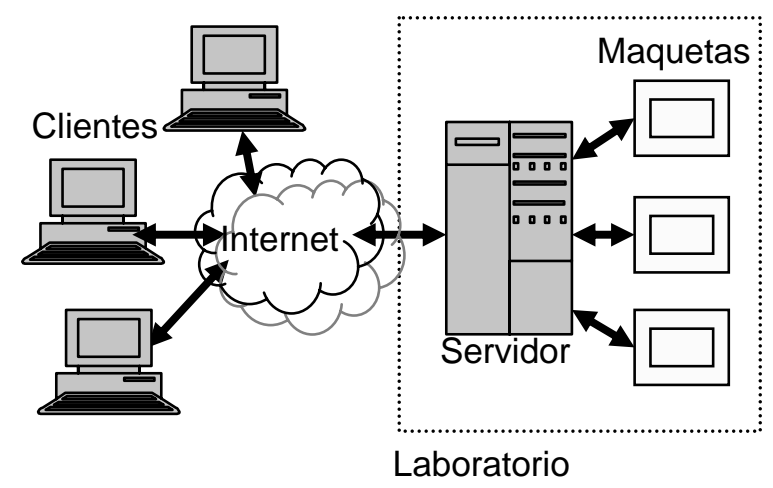

Fig.1: Arquitectura del sistema

El servidor de LABNET es una sola aplicación que se encarga de la gestión de los usuarios, de las maquetas y de la administración los experimentos. Por otra parte, el cliente de LABNET es la aplicación que permite el acceso remoto al laboratorio, y a través de su interfaz grafica, los usuarios pueden especificar sus experimentos de forma rápida y transparente.

\section{Modos de control}

El entorno de LABNET ofrece la posibilidad de realizar experimentos de control en lazo abierto o en lazo cerrado con controladores predefinidos que se pueden ejecutar de forma local o remota. En el modo local, el controlador está ubicado en el servidor. En este caso, el procesamiento de los experimentos es de tipo por lote "batch" y los resultados se visualizan una vez finalizados los experimentos. En la modalidad de control remoto (Overstreet y Tzes, 1999), el controlador se ejecuta en el cliente, por lo que la realimentación se hace a través de la red. Los controladores predefinidos de LABNET son las tres variantes más comunes del controlador PID: el PID teórico, el PID con filtrado de la derivada y el PID con Anti-Windup. Estas tres realizaciones recogen los aspectos prácticos más importantes de los PID industriales (Åström y Hagglund, 1995).

\section{Las maquetas}

Actualmente, están siendo utilizadas tres modelos de maquetas en el entorno de LABNET. La primera es una maqueta de control de nivel (Fig. 2a) y consiste en un circuito cerrado de dos tanques que se comunican mediante una bomba. Cuenta con dos sensores para medir el nivel del líquido y el caudal de la bomba. La segunda es una maqueta de control de temperatura y simula el sistema de calefacción de un recinto cerrado. (Fig. 2b). La maqueta cuenta con dos mecanismos para la simulación de perturbaciones; la apertura de una ventana y la configuración de la temperatura externa. Esta maqueta es construida a base de circuitos. La tercera es un sistema de estabilización de barcos (Fig. 2c) y consiste en un circuito de dos depósitos conectados por medio de una bomba reversible. Está equipado con dos sensores para medir la inclinación y la velocidad de giro, y cuenta también con dos mecanismos para la generación de perturbaciones; un generador de olas y varios discos magnéticos para modificar la distribución de la carga sobre el casco del barco.

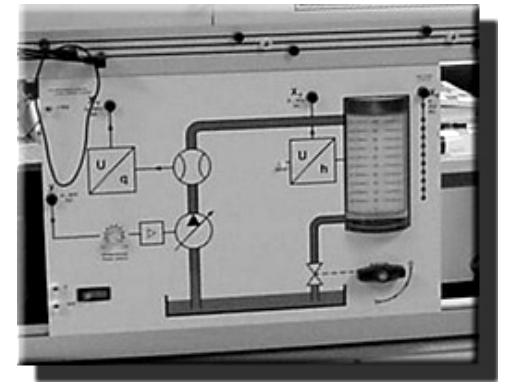

a) Control de nivel

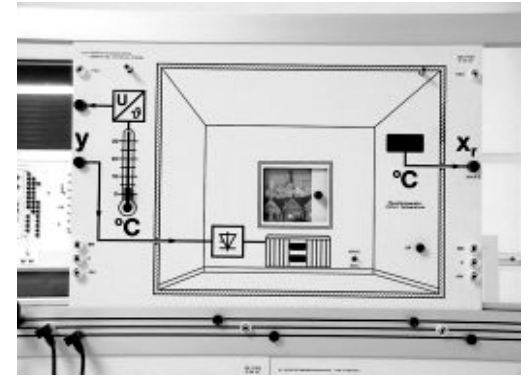

b) Control de temperatura

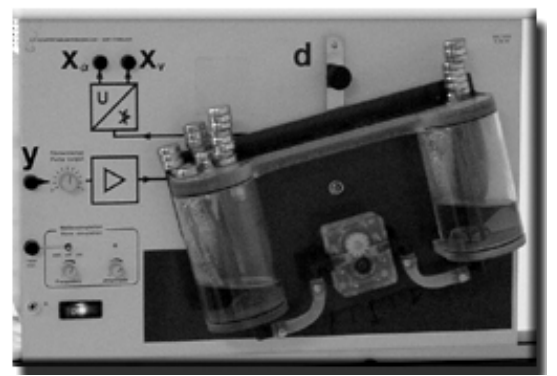

c) Estabilizador de barcos

Fig 2: Maquetas utilizadas en LABNET 


\section{Capacidad experimental de LABNET}

El entorno de LABNET permite diseñar una gama extensa de experimentos de control: control en lazo abierto o el lazo cerrado con controladores predefinidos como (P, PI, PD y PID) que se pueden ejecutar en lado del servidor o en el lado del cliente. Estas leyes de control se pueden experimentar sobre tres modelos de maquetas que tienen dinámicas diferentes. También se pueden realizar estudios del efecto del periodo de muestreo sobre el sistema de control, el efecto de los retardos de transmisión por la red sobre la realimentación, etc. Por último, LABNET ofrece la posibilidad de exportar los datos de los experimentos a los entornos de Matlab y de Excel, posibilitando así, un procesamiento de datos fuera de línea.

\section{EL CLIENTE}

El acceso remoto al laboratorio se hace a través del cliente de LABNET. Una sesión se inicia con un proceso de autenticación mediante un usuario y una clave. Una vez conectado al sistema, el usuario tiene acceso a todas las utilidades del sistema navegando por sistema de menús o mediante una barra de herramientas que permite un acceso rápido a todos los cuadros de dialogo necesarios en la realización de un experimento. El sistema de navegación es intuitivo y mucho cuadros de dialogo incorporan ayudas que proporcionan definiciones de conceptos o la explicación de ciertos procedimiento. El cliente cuenta con varias utilidades y las más importantes son la especificación de los experimentos, la visualización de datos e informes y la reutilización de plantillas.

\section{Especificación de experimentos}

El usuario puede especificar y personalizar sus experimentos. Al conectarse al sistema, el servidor informa de las maquetas disponibles. Después de la selección una maqueta, el usuario elige un controlador y configura sus parámetros, elige la señal de referencia, etc. Muchos otros parámetros como el periodo de muestro, el tiempo del experimento, y otros, tienen que configurarse antes de iniciar la ejecución del experimento. El usuario puede realizar todas estas especificaciones en cualquier orden o de forma secuencial, dejando el sistema desplegar los cuadros de dialogo necesarios para completar la especificación del experimento. La Fig. 3 muestra el aspecto de la interfaz gráfica del cliente así como unos cuadros de diálogo utilizados en la especificación de los experimentos.

\section{Visualización de datos e informes}

LABNET ofrece varias utilidades para el procesamiento de datos. La primera de ellas consiste en visualizar gráficamente las señales involucradas en los experimentos como las señales de referencias, la respuesta del sistema o la señal de control. La segunda permite a los usuarios exportar los datos de un experimento determinado a los entornos de Matlab o Excel. Una tercera utilidad consiste en la generación automática de informes de los experimentos. Estos informes son archivos estructurados que contienen datos de identificación del usuario, de las maquetas empleadas en el experimento, el controlador y el ajuste de sus parámetros, y contiene también una serie de enlaces a las gráficas y a los datos del experimento. Esta utilidad se ha mostrado de gran ayuda para los alumnos para documentar las prácticas de laboratorio. La Fig. 4, muestra un ejemplo de un informe generado de forma automática.

\section{Reutilización de plantillas}

La reutilización de plantillas constituye una alternativa para la especificación de los experimentos. Una plantilla es un modelo de un experimento previamente guardado que el usuario puede recuperar, realizar cambios eventuales en la especificación del experimento y ejecutarlo de forma inmediata.

Este mecanismo supone una simplificación significativa en el proceso de especificación de los experimentos. Una plantilla es un archivo de texto que contiene todos los parámetros utilizados en un experimento, por lo que los usuarios experimentados pueden realizar cambios en dicho archivo para definir sus experimentos haciendo uso de un simple editor de texto. 


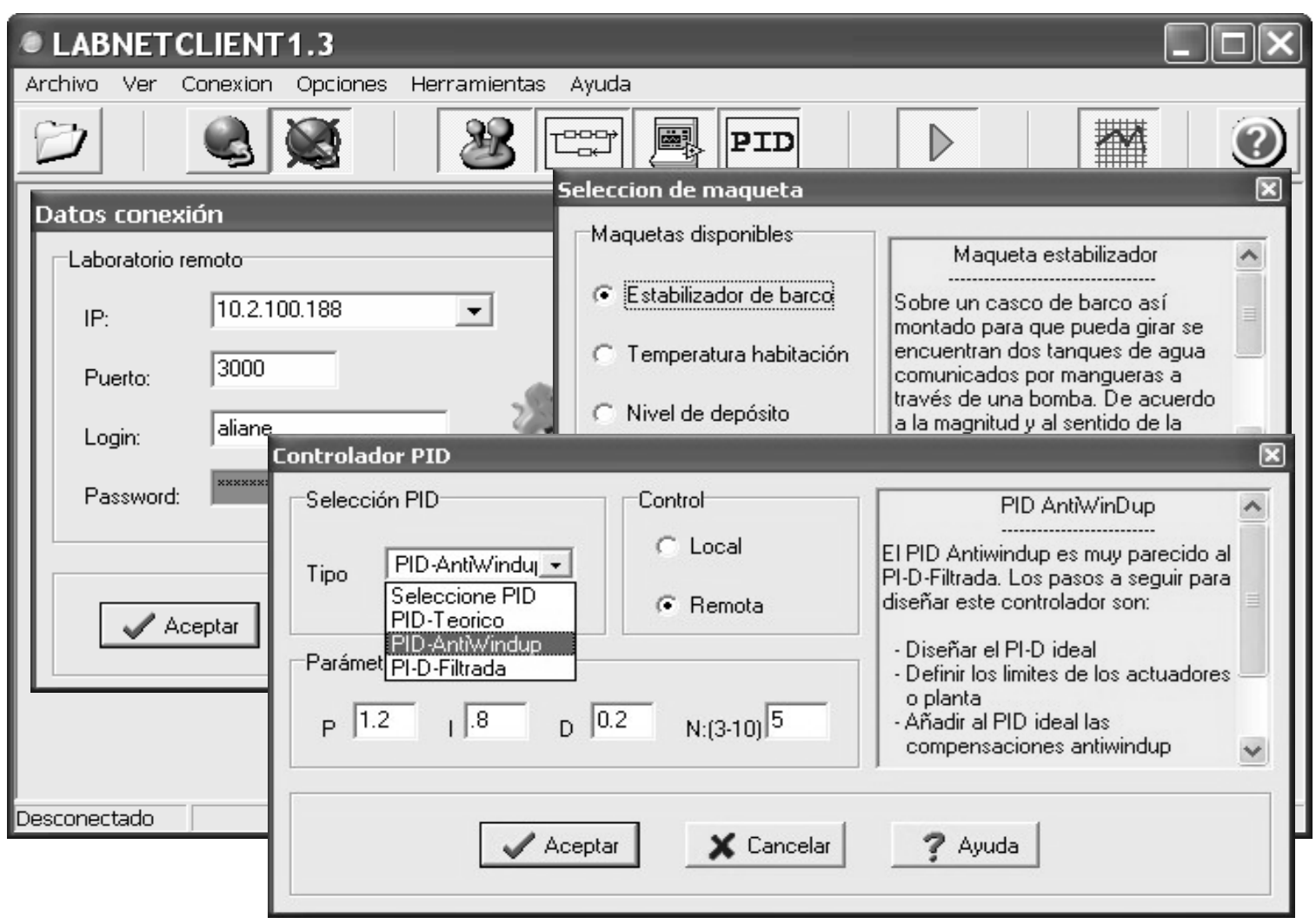

Fig. 3: El cliente LABNET y uno cuantos cuadros de diálogo

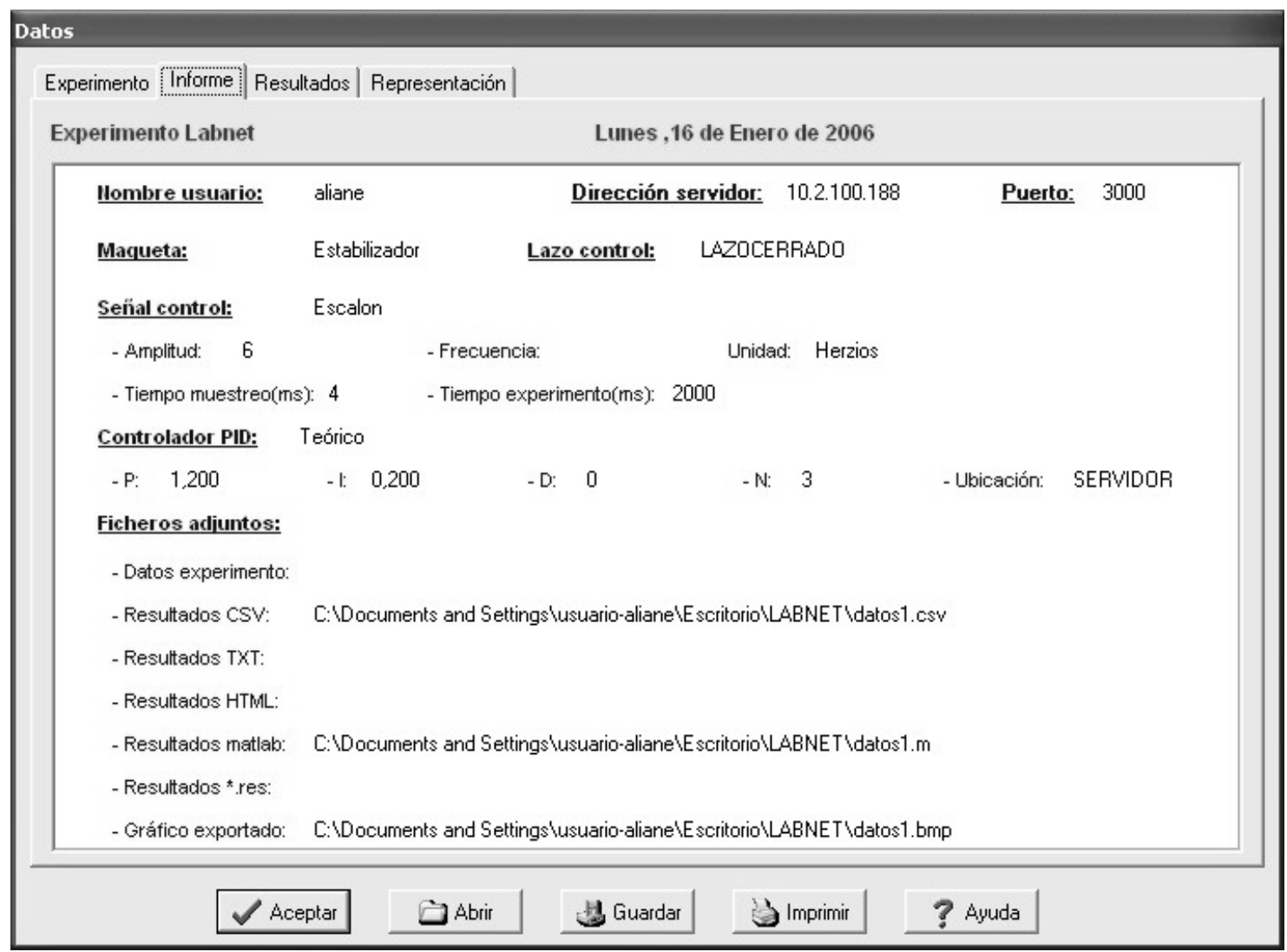

Fig. 4: Informe de un experimento 


\section{EL SERVIDOR}

El servidor de LABNET es una sola aplicación que tiene dos modos de funcionamiento: fuera de línea y en línea. En modo fuera de línea, el servidor ofrece los servicios relacionados con la gestión de los usuarios y la administración de las maquetas. Estas tareas de gestión son accesibles desde una interfaz gráfica sencilla como lo muestra la Fig. 5. Estando en modo en línea, el servidor se encarga de gestionar las solicitudes remotas y administrar los experimentos en tiempo real. El servidor tiene la posibilidad de monitorizar en tiempo real los experimentos en ejecución, identificando a los usuarios conectados, su punto de conexión (dirección ip), las maquetas utilizadas, el tipo de experimentos que está realizando, y muchos otros parámetros relativos al experimento que se está realizando.

\section{Administración de las maquetas}

Para facilitar la tarea de integración de las maquetas y la correspondiente tarea de asignación de las entradas-salidas, el diseño del servidor se ha basado en el empleo de modelos virtuales, que son objetos software que tienen todos los parámetros de las maquetas, como la polaridad o los niveles eléctricos de las señales involucradas en su control. El administrador realiza todas las conexiones de las maquetas al sistema guiado por una aplicación grafica intuitiva como lo muestra la Fig. 6. El usuario final solo ve las maquetas conectadas al sistema y no realiza ninguna operación de configuración.

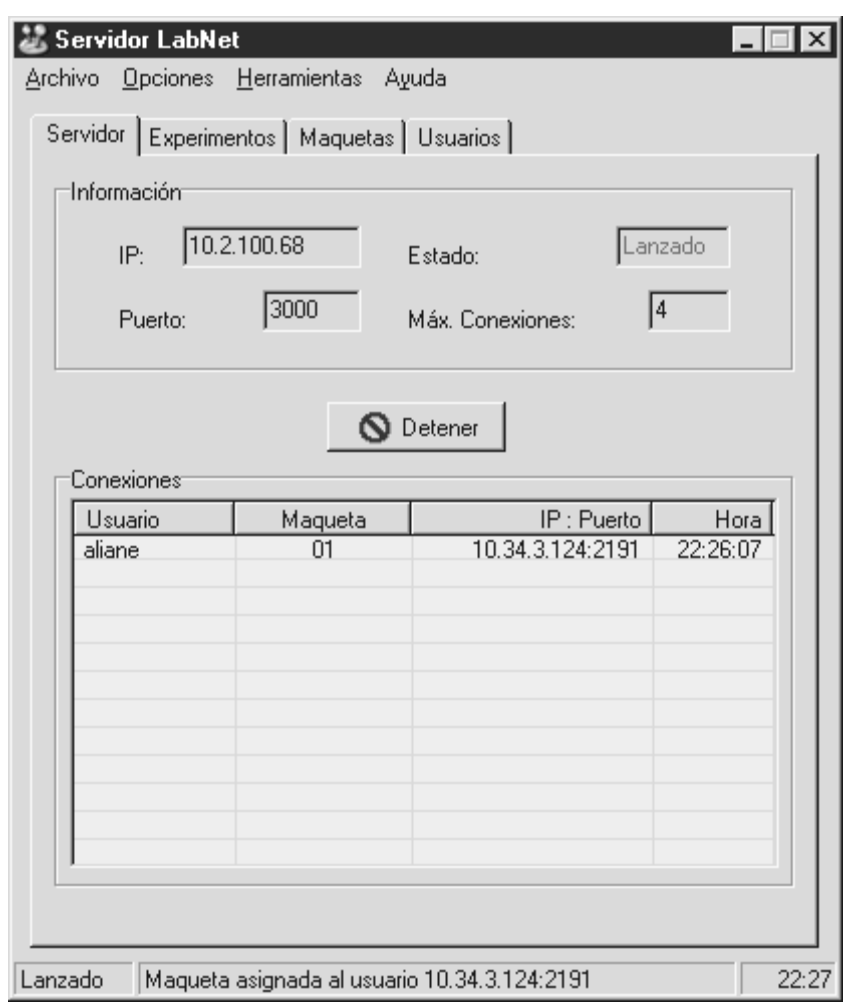

Fig. 5: El servidor LABNET

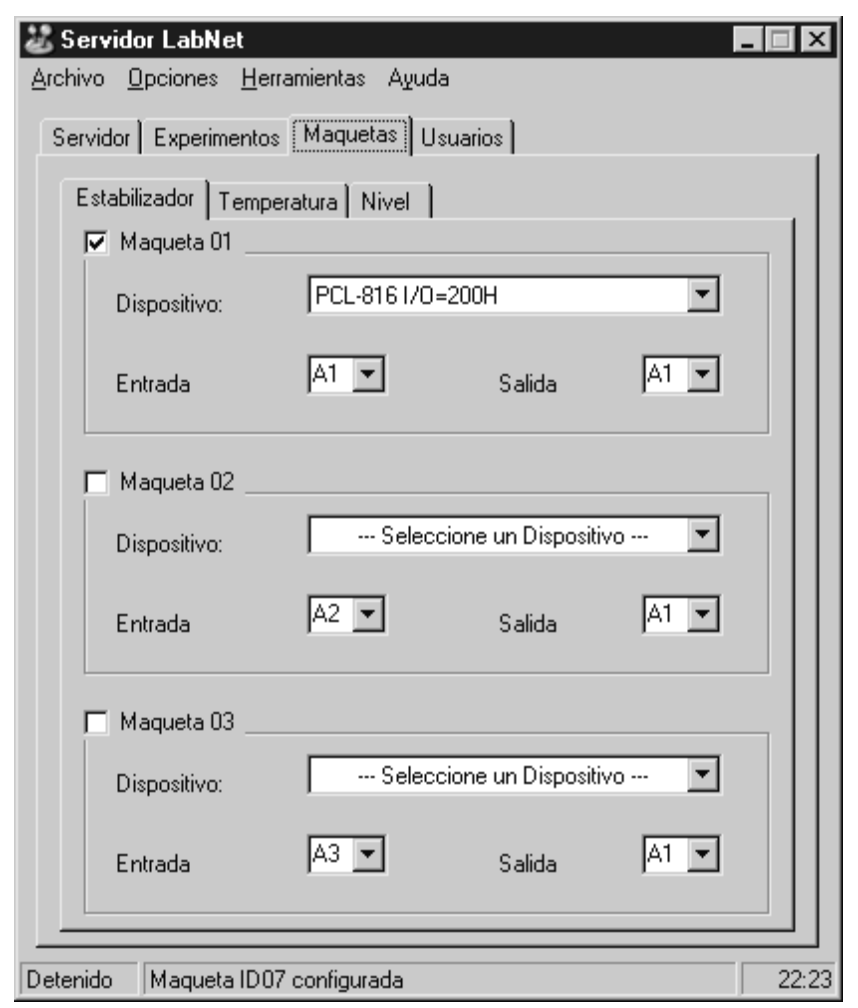

Fig. 6: Administrador de las maquetas

\section{CONSIDERACIONES SOBRE LA IMPLEMENTACIÓN}

El servidor de LABNET ha sido diseñado como una aplicación multi-hilo que puede atender de forma simultánea hasta cuatro clientes. El modelo de la concurrencia es el de un hilo por cliente (o sesión). Es decir, cuando se conecta un cliente y si queda alguna maqueta sin asignar, entonces el servidor crea un hilo independiente que atiende todas las solicitudes de dicho cliente. Este modelo permite a un usuario realizar varios experimentos durante una misma sesión. Las aplicaciones cliente y servidor han sido codificadas en $\mathrm{C}++$ haciendo uso de los hilos de la API Win32. 
La sincronización y la gestión del tiempo se han llevado a cabo haciendo uso del reloj multimedia de de la API de Windows. Este reloj permite alcanzar un periodo de muestreo de hasta cuatro milisegundos $(4 \mathrm{~ms})$, que es más que suficiente para implementar un control digital robusto para controlar las maquetas comentadas anteriormente.

La implementación digital de los todas las variantes de los controladores PID se ha obtenido mediante la reconversión del diseño analógico utilizando la aproximación de Tustin. Finalmente, para comprobar el funcionamiento correcto de los módulos, como el generador de señales, la implementación digital de los controladores, se han realizado varias pruebas comparativas con simulaciones e implementaciones realizadas sobre Matlab/Simulink.

\section{EVALUACIÓN DEL SISTEMA}

En el transcurso del curso pasado, se ha hecho una experiencia piloto enfocada a evaluar las diferentes funcionalidades de LABNET y recoger la opinión de los alumnos sobre su utilización. Esta experiencia consiste en la realización de dos prácticas de laboratorio utilizando opcionalmente el entorno remoto de LABNET. Solo el 68\% de los alumnos (17 alumnos de 25) optaron por la experimentación remota y el resto han preferido la experimentación real en laboratorio en presencia del profesor. Todas las conexiones al servidor se hicieron desde el Campus y el $53 \%$ de ellas se hicieron desde los ordenadores del propio laboratorio y en presencia del profesor.

Desde el punto de vista funcional, el $58 \%$ de los participantes llegaron a trabajar correctamente y sin asistencia durante más de 30 minutos en su primer intento, y el 23\% llegaron a registrar tiempos de trabajo continuado entorno a 60 minutos. Tan solo el $17 \%$ de los alumnos llegaron a trabajar sin asistencia. Las peticiones de asistencia, en general, han sido dudas entorno a conceptos de control y relativas al manejo de las maquetas. Las peticiones de asistencia relativas a la explotación de las utilidades del cliente han sido realmente pocas.

En general, los alumnos que optaron por la experimentación remota mostraron una actitud positiva y han manifestado que LABNET es muy intuitivo y fácil de usar. Varios alumnos confesaron haber tenido una experiencia muy interesante y que les ha permitido ampliar su visión sobre las posibilidades de Internet. Por otra parte, la mayor crítica ha sido en torno a la falta del sentido de presencia durante la experimentación y la falta de asistencia en línea.

\section{CONCLUSIONES}

En este artículo se ha presentado LABNET, un laboratorio remoto basado en Internet para la educación en la ingeniería de control. El laboratorio remoto ofrece la posibilidad de realizar una gama extensa de experimentos de control. El sistema puede dar servicio de forma concurrente hasta cuatro usuarios. Algunas utilidades como la reutilización de plantilla o la generación automática de informes, han resultado de gran interés, ya que han permitido darle mayor funcionalidad al sistema.

Las pruebas realizadas, desde el punto de vista de los usuarios, avalan la aceptación del sistema por parte de los alumnos. La experiencia adquirida, demuestra que un entorno de experimentación remota es factible y que los alumnos se adaptan rápidamente a un escenario educativo que implique la utilización de los laboratorios remotos.

El desarrollo de este primer prototipo abre un amplio abanico de posibles mejoras. Desde el punto de vista funcional, se está considerando dotar a LABNET de un sistema de gestión de reservas y de un sistema de benchmarking para comparar el desempeño de los diferentes controladores. Asimismo, se está contemplando incrementar su capacidad experimental con la incorporación de utilidades como la identificación en línea y el ajuste automático de los controladores. Estas mejoras se plasmarán en un segundo prototipo y harán que LABNET sea una herramienta educativa eficiente.

Finalmente, la utilización de un laboratorio remoto implica cambios sustanciales en las metodologías empleadas en la realización de los trabajos de laboratorio. En consecuencia, es necesario valorar su impacto pedagógico y la investigación en este campo debe enfocarse para generar pautas y orientaciones de como utilizar los laboratorios remotos para mejorar el aprendizaje. 


\section{REFERENCIAS}

Aburdene, M.F., E.J. Mastascusa y R. Massengale; A proposal for a remotely shared control systems laboratory, Proceedings of the ASEE, Frontiers in Education Conference, 589-592, USA-Lafayette, (1991)

Aktan, B. y C.A. Bohus; Distance Learning Applied to Control Engineering Laboratories, IEEE Transactions on Education, 39(3), 320-326 (1996).

Åström, K.J. y T. Hagglund; PID Controllers: Theory, Design, and Tuning, 2nd Edition, 59-116, ISA, North Carolina, USA (1995).

Aziz, E.S., S.K. Esche y C. Chassapis; A Scalable Platform for Remote and Virtual Laboratories, World Transactions on Engineering and Technology Education, 5(3), 445-448 (2006).

Berstein, D.S. y H. Ashrafioun; Innovation in Undergraduate Control Education, IEEE Control System Magazine, 24(5), 18-18 (2004).

Candelas, F.A. y J.M. Moreno; Recursos Didácticos Basados en Internet para el Apoyo a la Enseñanza de Material del Área de Ingeniería de Sistemas y Automática, Revista Iberoamericana de Automática e Informática Industrial, 2(2), 93-101 (2005).

Casini, M y D. Prattichizzo; The Automatic Control Telelab: A User-Friendly Interface for Distance Learning, IEEE Transaction on Education, 46(2), 252-257 (2003).

Casini, M, D. Prattichizzo y A. Vicino; The Automatic Control Telelab: A Web-based Tecnology for Distance Learning, IEEE Control Systems Magazine, 24(3), 36-44 (2004).

CEA-IFAC: Recopilación de laboratorios virtuales y/o remotos (en línea): http://www.ceaifac.es/wwwgrupos/educontrol/enl-labs.html, Acceso: 15 Junio (2007).

Cooper, M.;, Proyecto PEARL: Colección de artículos relacionados con los laboratorios remotos, (en línea) (2003), http://iet.open.ac.uk/pearl/publications/index.htm, Acceso: 15 Junio (2007).

Cooper, M.; Remote laboratories in teaching and learning: International Journal of Online Engineering, ISSN: 1861-2121, (en línea), 1(1) (2005), http://www.i-joe.org/ojs/include/getdoc.php? id=48\&article=11\&mode=pdf, Acceso: 15 Junio (2007).

CYBERLAB; Proveedor de servicios de experimentación remota (en línea) http://www.cyberlab.org, Acceso: 10 junio (2007).

Dormido, S.; "Control learning: Present and future", Annual Review in Control, 18(1), 115-136 (2004).

Esche, S.K; On the integration of remote experimentation into undergraduate laboratories: technical implementation. International Journal of Instructional Media, 33(1), 43-53 (2006).

Müller, D. y J.M. Ferreira; Online labs and the MARVEL experience, International Journal of Online Engineering, ISSN: 1861-2121, (en línea), 1(1) (2005), http://www.i-joe.org/ojs/include/getdoc.php? $\mathrm{id}=41 \&$ article $=4 \&$ mode $=$ pdf, Acceso: 15 Junio (2007).

Nickerson, J.V., J.E. Corter y S.K. Esche; A model for evaluating the effectiveness of remote engineering laboratories and simulations in education, Computers \& Education, 49(3), 708-725 (2007)

Overstreet J.W y A. Tzes; Real-Time Control Engineering Laboratory, IEEE Control Systems Magazine, October, 19(5), 19-34 (1999). 